\title{
Prioritizing Collision Avoidance and Vehicle Stabilization for Autonomous Vehicles
}

\author{
Joseph Funke ${ }^{1,2}$, Matthew Brown ${ }^{1}$, Stephen M. Erlien ${ }^{1}$, and J. Christian Gerdes ${ }^{1}$
}

\begin{abstract}
One approach to autonomous vehicle control is to generate and then track a desired trajectory without explicit consideration of vehicle stability. Stabilization is then entrusted to the vehicle's built-in production systems, such as electronic stability control, which constantly augment driving inputs to ensure stability. Other approaches explicitly consider stabilization criteria and implement permanently active constraints on the vehicle's actions. Situations exist, however, where enforcing stability constraints could lead to an otherwise avoidable collision. This paper presents an alternative paradigm for autonomous vehicle control that explicitly considers vehicle stability and environmental boundaries as it attempts to track a trajectory; such a mediator can choose to violate short term stability constraints in order to avoid a collision. Model predictive control provides an implementation framework, and an autonomous vehicle demonstrates the viability of the controller as it performs aggressive maneuvers. Driving around a turn at the vehicle's limits exhibits the importance of vehicle stability for autonomous vehicle control. Performing an emergency double lane change, however, highlights a situation where stability criteria must be temporarily violated to avoid a collision.
\end{abstract}

\section{INTRODUCTION}

As autonomous vehicles move from the research laboratory to public roads, it becomes necessary to consider how emergency scenarios-such as a deer jumping into the road or an unexpected patch of ice-push the vehicle beyond its handling limits. These scenarios can place competing demands of collision avoidance and vehicle stabilization on autonomous vehicles.

Research has focused on either vehicle stability or trajectory generation and tracking, but rarely both. Vehicle stability algorithms are implemented in production cars with systems such as electronic stability control (ESP) [1] and are studied academically using alternative approaches such as phase plane analysis [2], [3]. Meanwhile, autonomous vehicle control blossomed after the DARPA Urban Challenge. Boss and Junior, top competition finishers, operated by first planning and then tracking a desired trajectory [4], [5]. Since then autonomous vehicle research has made great advances toward real-world driving capabilities while maintaining this trajectory generation/tracking paradigm [6], [7]. These approaches do not explicitly consider vehicle stability, as vehicle operation is assumed to be below the handling limits; they presumably rely on the vehicle's production stability systems such as ESP, should such a scenario arise.

Model predictive control (MPC) provides an alternative framework in which an optimization continuously resolves to minimize path tracking error only within vehicle stability

\footnotetext{
${ }^{1}$ Department of Mechanical Engineering at Stanford University, USA.

${ }^{2}$ This is the corresponding author. jfunkelstanford.edu
}

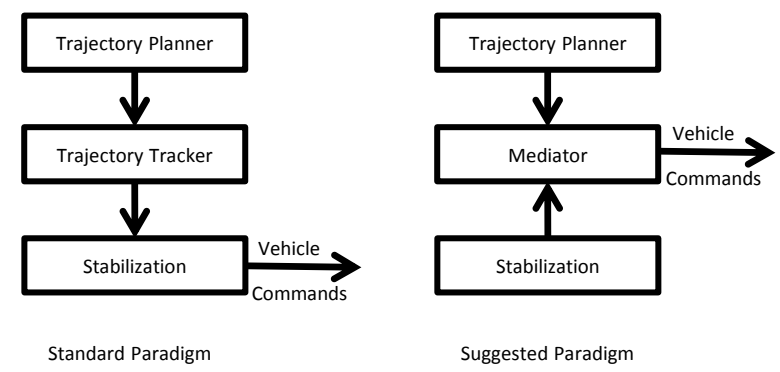

Fig. 1. Paradigms for autonomous vehicle control

constraints. Turri et al. [8] and Falcone et al. [9] implemented MPC frameworks that can avoid an obstacle in icy conditions. Turri's controller enforces stability by limiting tire slip angles to within the linear, non-saturated region. Falcone discusses a stability law more generally applicable to nonlinear systems and compares this criteria to the more vehicle-specific tire slip angle limits.

Implicitly or explicitly, all of these controllers incorporate permanently active vehicle stabilization. While usually beneficial, limiting the vehicle's actions for the sake of stabilization can sometimes lead to an otherwise preventable collision. The main contribution of this paper is an integration of collision avoidance and vehicle stabilization into one controller that can choose to violate stability criteria to prevent collision. In this paradigm, pictured in Fig. 1 , a controller resides between trajectory generation and stabilization and mediates between sometimes conflicting objectives by enforcing a hierarchy of importance: collision avoidance, then vehicle stability, and finally path tracking. This integration is achieved by reformulating Erlien et al.'s MPC driver assistance steering system [10] for autonomous vehicle control and incorporating longitudinal control. This paper also demonstrates through an experiment on a real vehicle that temporarily violating stability constraints can allow a vehicle to avoid a collision.

The paper begins by introducing the trajectories used in these examples, followed by a description of the vehicle model used in the controller. Vehicle states must remain inside an environmental envelope to avoid collisions and inside a stability envelope to enforce stabilization. These envelopes are introduced and then the full longitudinal and lateral controller is explained. Finally, two experiments are discussed. In the first, an autonomous vehicle drives around 
a turn at its handling limits with imperfect knowledge of the road surface, demonstrating the controller's ability to safely control the vehicle by enforcing the stability envelope. In the second, the vehicle successfully avoids an obstacle in an emergency lane change maneuver, but only by temporarily violating the stability envelope.

\section{TRAJECTORY}

Trajectories define the path and speed for the autonomous vehicle to follow in the absence of stability and environment constraints. This controller incorporates trajectories defined in terms of a path and speed profile.

\section{A. Path}

The path provides a nominal route for the vehicle to follow and a reference for the vehicle model and environmental bounds. Paths are parameterized by curvature $K$ as a function of distance $s$ along the path. Given a starting East/North position $\left(E_{0}, N_{0}\right)$ and heading orientation $\psi_{0}$, the position and tangent direction along the path can be calculated as a function of curvature and path distance:

$$
\begin{aligned}
\psi(s) & =\int_{0}^{s} K(x) d x+\psi_{0} \\
E(s) & =\int_{0}^{s} \cos \psi(x) d x+E_{0} \\
N(s) & =\int_{0}^{s} \sin \psi(x) d x+N_{0}
\end{aligned}
$$

\section{B. Speed Profile}

The speed profile defines the desired vehicle speed $U_{x, \text { des }}$ at each distance $s$ along the path. Trade-offs between collision avoidance, stabilization, and path tracking become most apparent when tracking a path near the handling limits of the vehicle; a speed profile can be defined in terms of these limits.

Handling limits derive from the available road-tire friction $\mu$, which limits a vehicle's lateral acceleration $a_{y}$ and longitudinal acceleration $a_{x}$ to

$$
\sqrt{a_{x}^{2}+a_{y}^{2}} \leq \mu g
$$

where $g$ is the gravitational constant. Lateral acceleration $a_{y}$ relates to path curvature $K$ using a steady state assumption,

$$
a_{y} \approx U_{x}^{2} K
$$

limiting the speed along a path. Given a desired friction usage $\mu_{d e s}$, setting (4) to an equality and incorporating (5) yields speeds along the path corresponding to desired vehicle accelerations of $\mu_{\text {des }} g$ [11].

Setting $\mu_{\text {des }}$ equal to the actual road-tire friction creates a speed profile at the handling limits of the vehicle. Such a speed profile is used in the first example so that the vehicle drives around a turn at its handling limits. The speed profile is set constant for the second example, so that the vehicle cruises at a set speed for the emergency lane change.

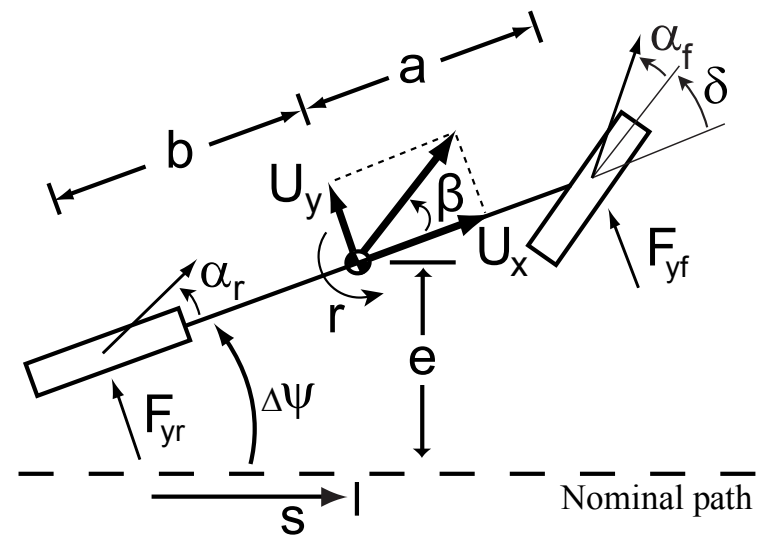

Fig. 2. Bike model schematic

\section{VEHICLE MODEL}

The MPC framework presented in the following sections represents the vehicle as a five state bicycle model. The first two vehicle states relate to vehicle stabilization, while the last three path states relate to path tracking and collision avoidance. Linearizing the governing equations produces an affine vehicle model, which enables real-time implementation.

\section{A. Vehicle States}

The vehicle is modeled using a planar bicycle model, shown in Fig. 2, in which the individual tires are combined into single, lumped front and rear tires. The velocity states, lateral velocity $U_{y}$ and yaw rate $r$, are described by the governing equations

$$
\dot{U}_{y}=\frac{F_{y f}+F_{y r}}{m}-r U_{x} \quad \dot{r}=\frac{a F_{y f}-b F_{y r}}{I_{z z}}
$$

where $F_{y f}$ and $F_{y r}$ are front and rear tire forces, $U_{x}$ is the vehicle's longitudinal velocity, $m$ is the vehicle's mass, $I_{z z}$ is the vehicle's moment of inertia, and $a$ and $b$ are distances from the vehicle's center of gravity to the front and rear axles respectively.

Tire forces $F_{y f}$ and $F_{y r}$ are defined as a function of maximum available force and tire slip angle $\alpha$. Slip angle is the angle between the tire's orientation and direction of travel,

$$
\begin{aligned}
& \alpha_{f}=\tan ^{-1}\left(\frac{U_{y}+a r}{U_{x}}\right)-\delta \approx \frac{U_{y}+a r}{U_{x}}-\delta \\
& \alpha_{r}=\tan ^{-1}\left(\frac{U_{y}-b r}{U_{x}}\right) \approx \frac{U_{y}-b r}{U_{x}}
\end{aligned}
$$

where $\delta$ is the steering angle. Tire forces are defined by a variant of the Fiala tire model [12],

$$
\begin{aligned}
F_{\mathrm{y}} & =\left\{\begin{array}{cc}
-C_{\alpha} \tan \alpha+\frac{C_{\alpha}^{2}}{3 \eta \mu F_{z}}|\tan \alpha| \tan \alpha \ldots \\
-\frac{C_{\alpha}^{3}}{27 \eta \mu^{2} F_{z}^{2}} \tan ^{3} \alpha, & |\alpha|<\alpha_{\text {sat }} \\
-\eta \mu F_{z} \operatorname{sgn} \alpha, & \text { otherwise }
\end{array}\right. \\
& =f_{\text {tire }}(\alpha)
\end{aligned}
$$


where $F_{z}$ is the normal load, $C_{\alpha}$ and $\mu$ are experimentally determined tire cornering stiffness and friction coefficients, and $\alpha_{\text {sat }}$ is the saturating slip angle, which is defined as

$$
\alpha_{s a t}=\tan ^{-1}\left(\frac{3 \eta \mu F_{z}}{C_{\alpha}}\right)
$$

A derating factor $\eta$ has been added to the model to capture the reduced lateral force capability due to longitudinal tire force $F_{x}$. Friction limits (4) reduce the maximum achievable lateral force $F_{y}$ for a given longitudinal force $F_{x}$. This effect is modeled using a derating factor defined as follows:

$$
\eta=\frac{\sqrt{\mu^{2} F_{z}^{2}-F_{x}^{2}}}{\mu F_{z}}
$$

The front and rear tire models are encoded separately into the affine vehicle model. For the rear tires, (9) is linearized about predicted operating points to incorporate locally-accurate linear tire models. For the front tires, the controller directly controls lateral force through the steering input, so $F_{y f}$ directly serves as the input to the vehicle model. Once calculated, a desired $F_{y f}$ can be converted to a steering angle according to (7) and (9),

$$
\delta=\frac{U_{y}+a r}{U_{x}}-f_{\text {tire }}^{-1}\left(F_{y f}\right)
$$

where $f_{\text {tire }}^{-1}$ represents the inverse tire curve.

\section{B. Path States}

The last three states relate the vehicle to the path. Distance along the path $s$, heading error between the vehicle and path $\Delta \psi$, and lateral error from the path $e$ are described by the governing equations,

$$
\begin{aligned}
\Delta \dot{\psi} & =r-U_{x} K(s) \\
\dot{e} & =U_{x} \sin \Delta \psi+U_{y} \cos \Delta \psi \approx U_{x} \Delta \psi+U_{y} \\
\dot{s} & =U_{x} \cos \Delta \psi-U_{y} \sin \Delta \psi \approx U_{x}-U_{y} \Delta \psi \approx U_{x}
\end{aligned}
$$

assuming small $\Delta \psi$.

The resulting vehicle model can be expressed in continuous form with the affine expression,

$$
\dot{x}=A x+B F_{y f}+C
$$

where $x=\left[\begin{array}{lllll}U_{y} & r & \Delta \psi & s & e\end{array}\right]^{T} . A, B$, and $C$ depend on $U_{x}, \alpha_{r}$, and $K(s)$, which can vary at discrete time steps.

\section{STABILITY AND ENVIRONMENTAL ENVELOPES}

Vehicle stabilization and collision avoidance are defined in terms of envelopes.

\section{A. Stability Envelope}

The vehicle stability envelope defines a region of vehicle states in which the vehicle will remain stable. This controller uses the envelope suggested by Beal and Gerdes [3]. Their envelope bounds the vehicle's velocity states $r$ and $U_{y}$ by the maximum steady state force generation of the tires. In steady state, the maximum force generated from the front and rear tires is $m g \mu$, where $g$ is the gravitational constant and $\mu$ is the friction coefficient between the tires and road. Yaw rate $r$ is bounded based on the steady-state condition of (6) to yield a maximum yaw rate:

$$
r_{\max }=\frac{g \mu}{U_{x}}
$$

Lateral velocity $U_{y}$ is bounded by rear tire saturation. This occurs at the rear saturation slip angle (10) and can be converted using (8) to a bound on $U_{y}$ :

$$
U_{y \max }=U_{x} \alpha_{r, s a t}+b r
$$

The resulting envelope bounds vehicle velocity states $r$ and $U_{y}$ to an invariant set. Vehicle stability is guaranteed as long as these states remain in the envelope. Exceeding the envelope does not necessarily result in immediate instability, but robust stability guarantees are lost.

\section{B. Environmental Envelope}

Collision avoidance is modeled using an environmental envelope, as originally proposed by Erlien et al. [10]. The environmental envelope is a set of lateral error bounds $e_{\max }$ and $e_{\min }$ as a function of distance $s$ along a path that avoids obstacles and remains on the road. Enforcing these bounds on the vehicle's lateral error as follows,

$$
\begin{aligned}
& e^{k}+f_{\text {width }}\left(\Delta \psi^{k}\right) \leq e_{\text {max }}^{k} \\
& e^{k}-f_{\text {width }}\left(\Delta \psi^{k}\right) \geq e_{\text {min }}^{k}
\end{aligned}
$$

provides a convex method of enforcing environmental constraints on the vehicle's position. The function $f_{\text {width }}(\Delta \psi)$ linearly approximates the vehicle's effective width as its orientation from the path changes. For example, at $\Delta \psi=0$, the vehicle's effective width is half its actual width, while at $\Delta \psi=90^{\circ}$, the vehicle's effective width is $a$, the distance from the center of gravity to the front axle.

\section{CONTROLLER FRAMEWORK}

MPC lends itself well to a paradigm which trades off between sometimes conflicting objectives. In order to reliably solve the MPC optimization problem in real-time, the structure is limited to a convex form. Optimizing throttle and brake inputs as part of the MPC controller makes the problem nonconvex and difficult to solve in real-time [13]. Instead, the throttle and brake inputs are determined first and inform the MPC optimization for steering commands, incorporating the coupling between lateral and longitudinal dynamics [14].

\section{A. Longitudinal Controller}

The longitudinal controller assumes longitudinal dynamics based on a point mass and tracks the speeds defined by the speed profile. At each point in the MPC prediction horizon, the following feedforward plus feedback control law is used,

$$
F_{x, c m d}=m A_{x, \text { des }}(s)+k_{p}\left(U_{x, \text { des }}(s)-U_{x}\right)+F_{x, \text { drag }}
$$

where $U_{x, d e s}(s)$ is the speed of the speed profile at $s$, $A_{x, \text { des }}(s)$ is the necessary longitudinal acceleration to track the change in speed in the speed profile at $s, k_{p}$ is a speed tracking gain, and $F_{x, d r a g}$ offsets dissipative effects such as 
rolling resistance and aerodynamic drag. The commanded forces $F_{x, c m d}$ are calculated at each point in the prediction horizon and integrated to estimate vehicle speed throughout the horizon. The first $F_{x, c m d}$ in the horizon is converted to brake pressures and motor torques and applied to the vehicle.

\section{B. Lateral Controller}

MPC determines front tire forces based on a dynamic model of the vehicle. The affine vehicle model (16) is discretized into $n=30$ steps along a receding horizon, the most the solver can compute in real-time. The first $k=1 \ldots 10$ steps are discretized at $10 \mathrm{~ms}$, which is short enough to accurately capture the vehicle dynamics in the near term. Because the controller itself operates at $10 \mathrm{~ms}$ time steps, the vehicle model is discretized with a zeroorder hold to accurately reflect a constant steering input over the duration of a time step. The next $k=11 \ldots 30$ time steps are discretized at a much longer $200 \mathrm{~ms}$ in order to extend the prediction horizon further into the future without adding the computational complexity of hundreds of short time steps. Extending the horizon incorporates constraints such as obstacles further in the future so that the controller can plan accordingly. These time steps are discretized with a first-order hold to better approximate the steering control inputs over the long time step. Erlien et al. discuss the general two time step approach [10].

In the addition to the time step variation, the vehicle model at each step $k$ is also linearized around an expected operating point. Expected speeds $U_{x}$ and longitudinal inputs $F_{x}$ from the longitudinal controller, path curvature from expected positions along the path $K(s)$, and expected rear slip $\alpha_{r}$ from the previous MPC solution are used to create a timevarying, affine model used in the MPC optimization:

$x^{k+1}= \begin{cases}A^{k} x^{k}+B_{1}^{k} F_{y f}^{k}+C^{k} & k=0 \ldots 9 \\ A^{k} x^{k}+B_{1}^{k} F_{y f}^{k}+B_{2}^{k} F_{y f}^{k+1}+C^{k} & k=10 \ldots 29\end{cases}$

Matrices $A^{k}, B_{1}^{k}, B_{2}^{k}$, and $C^{k}$ are the linearized and discretized approximations of (16) at each time step $k$.

Stability constraints (17) and (18) can be represented in the MPC problem as

$$
H_{v e h}^{k} x^{k} \leq G_{v e h}^{k} \quad k=1 \ldots 30
$$

and the environmental envelope (19) is expressed as

$$
H_{e n v}^{k} x^{k} \leq G_{e n v}^{k} \quad k=11 \ldots 30
$$

Rather than weighting $F_{y f}$ to reduce the maximum steering angle, the optimization weights the change in input, $v^{k}=F_{y f}^{k}-F_{y f}^{k-1}$, in order to reduce steering changes. The final optimization takes the form

$$
\begin{aligned}
\min & \sum_{k=1}^{30}\left(x^{k}\right)^{T} Q^{k} x^{k}+\left(v^{k}\right)^{T} R^{k} v^{k}+\left(\sigma_{\text {veh }}^{k}\right)_{+} W_{v e h}^{k}+\ldots \\
& \sum_{k=11}^{30}\left(\sigma_{\text {env }}^{k}\right)^{T} W_{\text {env }}^{k} \sigma_{\text {env }}^{k}
\end{aligned}
$$

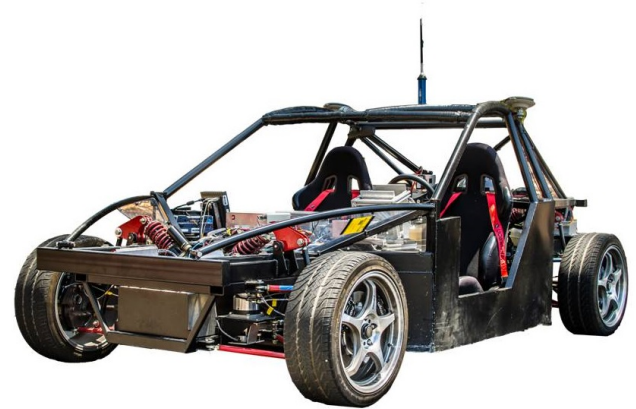

Fig. 3. Steer-by-wire test vehicle X1

subject to the vehicle model (21) and

$$
\begin{array}{rlrl}
H_{v e h}^{k} x^{k} & \leq G_{v e h}^{k}+\sigma_{v e h}^{k} & & k=1 \ldots 30 \\
H_{e n v}^{k} x^{k} & \leq G_{\text {env }}^{k}+\sigma_{\text {env }}^{k} & & k=11 \ldots 30 \\
\left|F_{y f}^{k}\right| & \leq \eta^{k} \mu F_{z f} & k & =0 \ldots 30 \\
\left|v^{k}\right| & \leq v_{\text {max }}^{k} & k & =1 \ldots 30
\end{array}
$$

where $\sigma_{v e h}$ and $\sigma_{e n v}$ are slack variables on the constraints enforcing the stability and environmental envelopes. Ideally, both constraints are met and the slack variables are zero. However, these soft constraints ensure problem feasibility if constraints must be violated, and setting $W_{\text {env }} \gg W_{v e h} \gg$ $\|Q\|_{\infty}$ encodes a prioritization of collision avoidance, then stability, and finally path tracking. Normalized weights are each one order of magnitude greater than the next to ensure this prioritization. Linear rather than quadratic weighting of $\sigma_{v e h}$ provides quicker response to small stability envelope violations and weights large violations relatively less in favor of minimizing collision costs. To enforce path tracking, the diagonal entries in $Q$ corresponding to lateral error $e$ and heading error $\Delta \psi$ are nonzero. As is standard with MPC, this optimization problem is resolved each time step, and $F_{y f}^{0}$ is converted to a steering angle command. The optimization is solved in real-time by code generated with CVXGEN [15].

\section{EXPERIMENTAL RESULTS}

Experiments are conducted with $\mathrm{X} 1$, a rear-wheel drive electric vehicle, shown in Fig. 3. A GNSS-aided Inertial Navigation System provides vehicle states in real-time, and a ruggedized i7 computer outputs controller commands at $100 \mathrm{~Hz}$ to the vehicle's drive-by-wire system.

\section{A. Enforcing Stability Criteria}

In the first scenario, the vehicle drives around a turn at its handling limits, a situation that could occur in some emergency scenarios or while racing. A speed profile is generated based on a desired friction utilization of $\mu_{\text {des }}=0.80$, slightly below the vehicle's steady state friction capability of $\mu=0.85$. However, the surface has a $3^{\circ}$ grade not accounted for in the speed profile generation. The desired speeds and actual tracking, at least within the acceleration capability of the motor, are plotted in Fig. 4. The road bounds, desired path, and surface grade are shown in Fig. 5. 


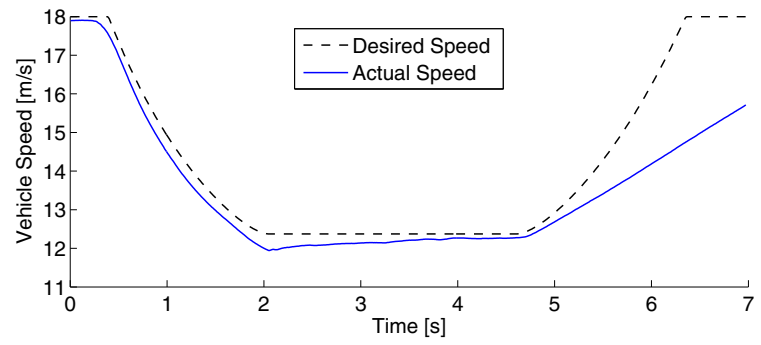

Fig. 4. Desired and actual vehicle speeds while turning at the limits

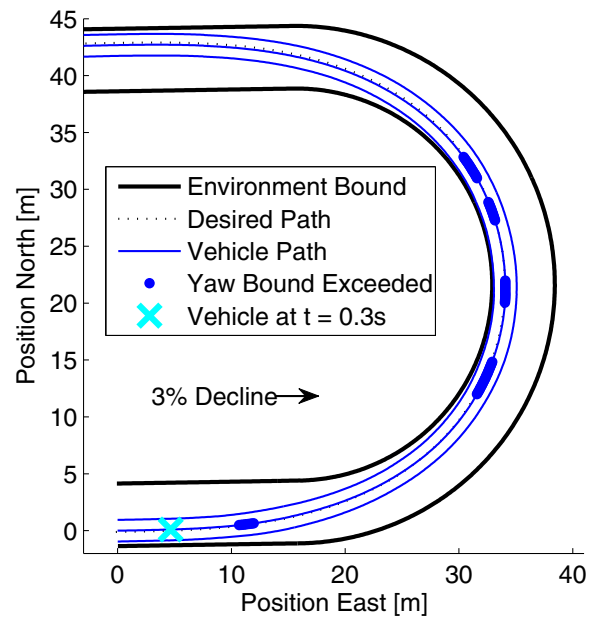

Fig. 5. Path and road bounds for turning at the limits

Figure 6 depicts the steering input, yaw rate, and lateral error as the vehicle drives around the corner. The solid dots highlight periods of time when the raw rate exceeds the yaw stability bound (17), and the open circles depict the MPC plan over the prediction horizon when at $t=0.3 \mathrm{~s}$.

Of the two vehicle velocity states $r$ and $U_{y}$, only yaw rate $r$ and its associated stability bound (17) are plotted in Fig. 6 because the $U_{y}$ stability constraint (18) is never active. The vehicle's yaw rate exceeds (17) several times, highlighted by the solid dots. The MPC plan at $t=0.3 \mathrm{~s}$, in open circles, does not predict exceeding the stability envelope, since the MPC model and trajectory are calculated assuming zero grade. Other factors could be ascribed to such a mismatch, but in this case the nonzero grade strongly contributes to this difference through $t=2-5 s$. The $3^{\circ}$ decline results in an off-camber turn of up to $3^{\circ}$ at the middle of the turn. The decreased normal force and additional lateral force theoretically reduces the effective friction to $\mu \approx 0.85 \cos 3^{\circ}-\sin 3^{\circ}=0.80$, the desired friction used to generate the speed profile. The first envelope violation at $t=0.75 \mathrm{~s}$ occurs during combined heavy braking and steering, resulting in a highly transient maneuver that challenges the simplifying assumptions of the controller.

Despite a trajectory designed to keep the vehicle within its limits, other factors difficult to know a priori still threaten
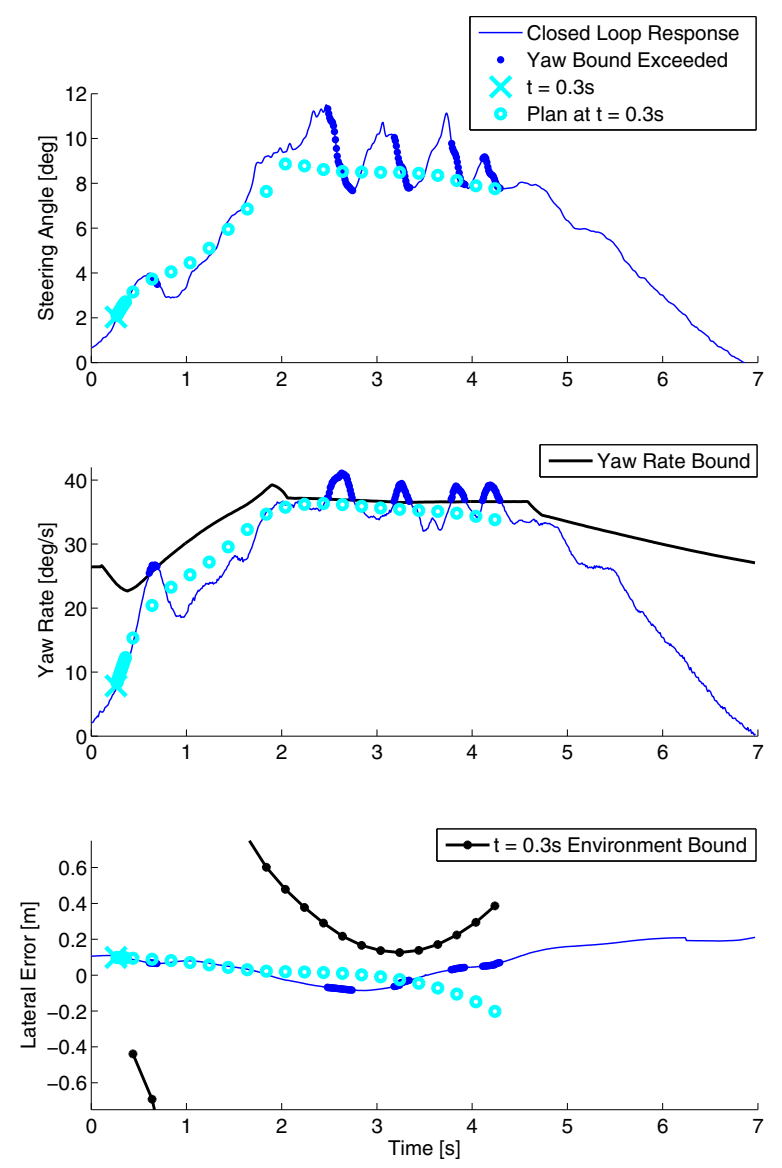

Fig. 6. Steering angle, yaw rate, and lateral error through the turn

vehicle stability. The controller responds by countersteering, as seen by the decreasing steering angle associated with each yaw rate bound violation. These corrections kept the violations small, and the lateral error remains below $25 \mathrm{~cm}$.

The vehicle is never in danger of running off the road, so the environmental envelope is never active and the stability envelope is continuously enforced. Also note that the maximum tracking error of $21 \mathrm{~cm}$ occuring at $t=7 \mathrm{~s}$, which is acceptable given the road bounds at that point, is unacceptable at $t=3 \mathrm{~s}$. These two times demonstrate the nonuniform importance of path tracking depending on the environmental envelope.

Ultimately, this situation highlights the importance of stability constraints to maintain control despite imperfect knowledge of the environment and the vehicle's capabilities.

\section{B. Violating Stability Criteria}

In the second scenario, the vehicle cruises at $16 \mathrm{~m} / \mathrm{s}$ along a straight path, defined as $K(s)=0 \forall s$, and detects an obstacle at $t=0 \mathrm{~s}$, necessitating the double lane change depicted in Fig. 7. The controller reacts to the obstacle detection immediately, steering to avoid the obstacle without running off the road. Figure 8 plots the resulting steering commands, yaw rate response, and lateral error. The MPC plan at $t=1 s$ is plotted in open circles as well. 


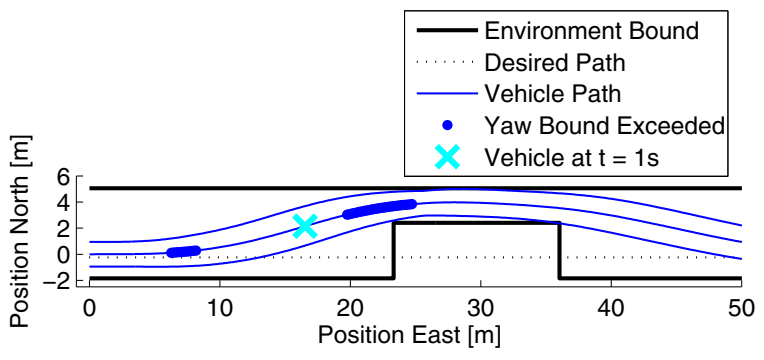

Fig. 7. Emergency double lane change
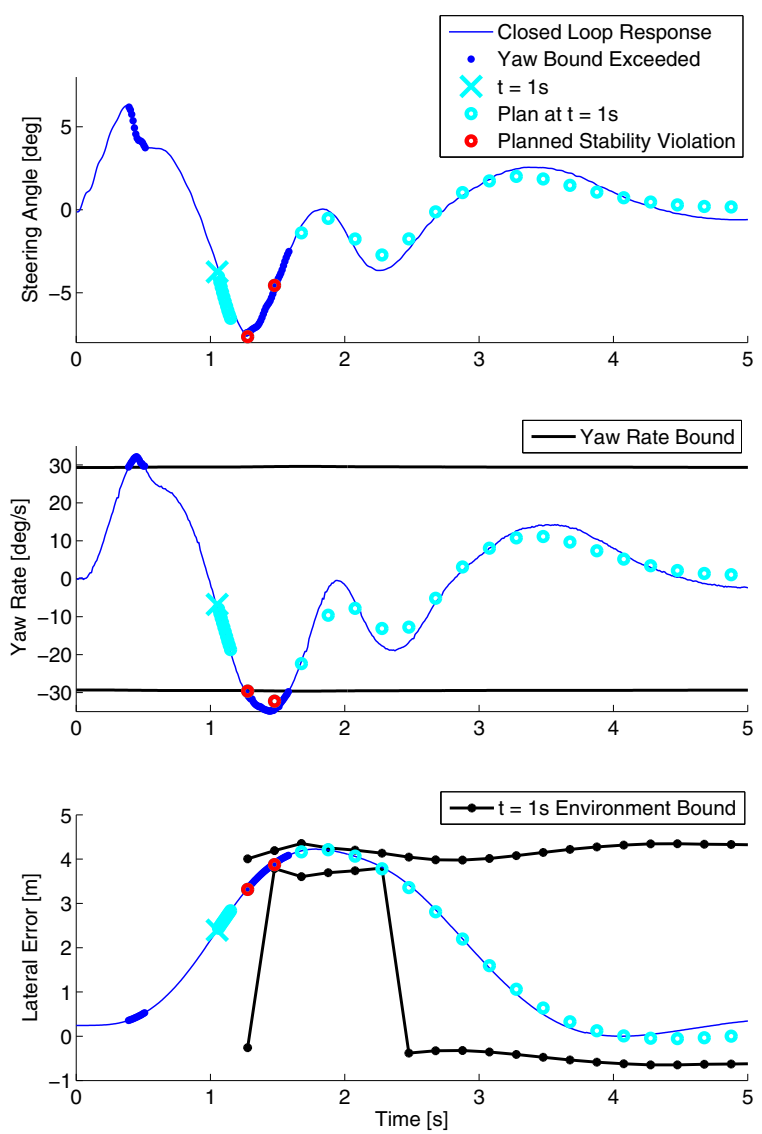

Fig. 8. Steering angle, yaw rate, and lateral error through the lane change

Environmental collision threatens, as the sides of the vehicle approach both the road edge and obstacle. Figure 7 shows this spatially, and Fig. 8 plots this as bounds on the vehicle's tracking error from the path. The lateral error bounds in Fig. 8 vary because they incorporate vehicle width and orientation according to $f_{\text {width }}\left(\Delta \psi^{k}\right)$ in (19). At $t=1 \mathrm{~s}$, the MPC plan places the vehicle at the environmental bounds. This collision-free path is possible only by planning to temporarily violate the yaw rate bound, as seen in red open circles. Exceeding this bound entails turning right faster than normally acceptable to avoid running off the left road edge. Because of this stability envelope violation, the vehicle passes by the obstacle without collision or road departure.
This scenario represents a situation where temporarily violating stability criteria allows the vehicle to avoid collision. This example, along with the previous scenario, together demonstrate the advantage of integrating and prioritizing collision avoidance and stabilization in one controller.

\section{ACKNOWLEDGMENTS}

This research is supported by the Electronics Research Laboratory of Volkswagen of America (ERL), with additional assistance from Robin Simpson and Rick Keiner. The authors would like to thank Thomas Lipp for convex optimization consultation, John Kegelman for the GPS setup, OmniSTAR for GPS corrections, and Master Metal Products for fabrication assistance. Testing was possible with help from Thunderhill Raceway Park. Funke and Brown were supported by Graduate Research Fellowships from the National Science Foundation.

\section{REFERENCES}

[1] E. Liebemann, K. Meder, J. Schuh, and G. Nenninger, "Safety and performance enhancement: The bosch electronic stability control (esp)," SAE Paper, vol. 20004, pp. 21-0060, 2004.

[2] S. Inagaki, I. Kshiro, and M. Yamamoto, "Analysis on vehicle stability in critical cornering using phase-plane method," in International Symposium on Advanced Vehicle Control, 1994.

[3] C. E. Beal and J. C. Gerdes, "Model predictive control for vehicle stabilization at the limits of handling," IEEE Transactions on Control Systems Technology, vol. 21, no. 4, pp. 1258-1269, 2013.

[4] C. Urmson, J. Anhalt, D. Bagnell, C. Baker, R. Bittner, M. Clark, J. Dolan, D. Duggins, T. Galatali, C. Geyer et al., "Autonomous driving in urban environments: Boss and the urban challenge," Journal of Field Robotics, vol. 25, no. 8, pp. 425-466, 2008.

[5] M. Montemerlo, J. Becker, S. Bhat, H. Dahlkamp, D. Dolgov, S. Ettinger, D. Haehnel, T. Hilden, G. Hoffmann, B. Huhnke et al., "Junior: The stanford entry in the urban challenge," Journal of Field Robotics, vol. 25 , no. 9, pp. 569-597, 2008.

[6] J. Levinson, J. Askeland, J. Becker, J. Dolson, D. Held, S. Kammel, J. Z. Kolter, D. Langer, O. Pink, V. Pratt et al., "Towards fully autonomous driving: Systems and algorithms," in IEEE Intelligent Vehicles Symposium, 2011, pp. 163-168.

[7] J. Ziegler, P. Bender, T. Dang, and C. Stiller, "Trajectory planning for bertha-a local, continuous method," in IEEE Intelligent Vehicles Symposium Proceedings, 2014, pp. 450-457.

[8] V. Turri, A. Carvalho, H. Tseng, K. H. Johansson, and F. Borrelli, "Linear model predictive control for lane keeping and obstacle avoidance on low curvature roads," in IEEE Conference on Intelligent Transportation Systems, 2013, pp. 378-383.

[9] P. Falcone, F. Borrelli, H. E. Tseng, J. Asgari, and D. Hrovat, "Linear time-varying model predictive control and its application to active steering systems: Stability analysis and experimental validation," International journal of robust and nonlinear control, vol. 18, no. 8, pp. 862-875, 2008.

[10] S. Erlien, S. Fujita, and J. C. Gerdes, "Safe driving envelopes for shared control of ground vehicles," in Advances in Automotive Control, vol. 7, no. 1, 2013, pp. 831-836.

[11] J. Funke and J. C. Gerdes, "Simple clothoid paths for autonomous vehicle lane changes at the limits of handling," in ASME Dynamic Systems and Control Conference, 2013.

[12] E. Fiala, "Lateral forces on rolling pneumatic tires," Zeitschrift VDI, vol. 96, no. 29, pp. 973-979, 1954.

[13] P. Falcone, H. Eric Tseng, F. Borrelli, J. Asgari, and D. Hrovat, "Mpc-based yaw and lateral stabilisation via active front steering and braking," Vehicle System Dynamics, vol. 46, no. S1, pp. 611-628, 2008.

[14] R. Attia, R. Orjuela, and M. Basset, "Combined longitudinal and lateral control for automated vehicle guidance," Vehicle System Dynamics, vol. 52, no. 2, pp. 261-279, 2014.

[15] J. Mattingley and S. Boyd, "Cvxgen: a code generator for embedded convex optimization," Optimization and Engineering, vol. 13, no. 1, pp. 1-27, 2012. 